\title{
Photoluminescence properties of AIN homoepilayers with different orientations
}

\author{
A. Sedhain, ${ }^{1}$ N. Nepal, ${ }^{1}$ M. L. Nakarmi, ${ }^{1}$ T. M. Al tahtamouni, ${ }^{1}$ J. Y. Lin, ${ }^{2}$ H. X. Jiang,,${ }^{2, a)}$ \\ Z. Gu, ${ }^{3}$ and J. H. Edgar ${ }^{3}$ \\ ${ }^{1}$ Department of Physics, Kansas State University, Manhattan, Kansas 66506-2601, USA \\ ${ }^{2}$ Nano Tech Center and Department of Electrical and Computer Engineering, Texas Tech University, \\ Lubbock, Texas 79409, USA \\ ${ }^{3}$ Department of Chemical Engineering, Kansas State University, Manhattan, Kansas 66506-5102, USA
}

(Received 30 January 2008; accepted 19 June 2008; published online 29 July 2008)

\begin{abstract}
AlN homoepilayers and heteroepilayers were grown on polar $c$-plane and nonpolar $a$-plane and $m$-plane orientations of AlN bulk and sapphire substrates by metal organic chemical vapor deposition. A systematic comparative study of photoluminescence properties of these samples revealed that all AlN homoepilayers ( $c, a$ and $m$ planes) were strain free with an identical band gap of about $6.099(6.035) \mathrm{eV}$ at $10(300) \mathrm{K}$, which is about $42 \mathrm{meV}$ below the band gap of $c$-plane AlN heteroepilayers grown on sapphire. Also, nonpolar $a$-plane homoepilayers have the highest emission intensity over all other types of epilayers. We believe that $a$-plane AlN homoepilayers have the potential to provide orders of magnitude improvement in the performance of new generation deep UV photonic devices. (C) 2008 American Institute of Physics. [DOI: 10.1063/1.2965613]
\end{abstract}

AlN has a complete solid solubility with GaN (Ref. 1) and emerged as an important semiconductor material for applications of light emitters down to $200 \mathrm{~nm}$ (Ref. 2) and detectors in the deep ultraviolet (DUV) and extreme UV spectral region. ${ }^{3,4}$ Quantum wells (QWs) have been the device structure of choice for efficient III-nitride semiconductor based light emitters. ${ }^{5,6}$ Conventional nitride $c$-plane multiple QW structures generate fixed sheet charges at the interfaces due to the spontaneous and piezoelectric polarization. ${ }^{7-10}$ which induce internal electric fields, lead to carrier separation, and reduce the radiative recombination rate. Consequently, optoelectronic devices such as light emitting diodes and laser diodes based on heteroepitaxial $c$-plane oriented III-nitride materials possess reduced internal quantum efficiencies.

There has been tremendous effort in the investigation of nonpolar $a$-plane and $m$-plane III-nitride epilayers ${ }^{11}$ and optoelectronic devices with QW structures ${ }^{9,10}$ to reduce the effects of internal electric fields. To achieve QW and other heterostructure based devices with improved performance, optical properties of epilayers grown on different orientations of available substrates have to be better understood. Furthermore, most previous studies on the fundamental band structures of AlN have been carried out for heteroepilayers grown on sapphires, which are plagued by lattice mismatch induced strain and high threading dislocation density, which significantly inhibited our ability for precisely determining the fundamental band structure parameters of AlN. In this work, we report a systematic comparative study of optical properties of both homoepitaxial and heteroepitaxial layers of AlN grown on polar $c$-plane as well as $a$-plane and $m$-plane orientations probed by DUV photoluminescence (PL).

AlN bulk single crystal substrates were produced by sublimation crystal growth using polycrystalline AlN wafer as seeds and have a thickness of about $1 \mathrm{~mm}$ and an average

${ }^{a)}$ Electronic mail: hx.jiang@ttu.edu. grain size of about $2 \times 3 \mathrm{~mm}^{2}{ }^{12}$ The surface of AlN bulk crystal substrates was prepared by chemical mechanical polishing (done by NovaSiC) that provided a surface roughness of about 1-4 $\mathrm{nm}$. The dislocation density in AlN bulk single substrates was below $1 \times 10^{6} \mathrm{~cm}^{-2}$ as determined by synchrotron white beam x-ray topography. ${ }^{13}$ The $1 \mu \mathrm{m}$ thick undoped AlN epilayers were grown on $c$-plane, $a$-plane, and $m$-plane bulk AlN and $c$-plane and $r$-plane sapphire substrates by metal organic chemical vapor deposition (MOCVD). A low temperature AlN nucleation layer was deposited at $500{ }^{\circ} \mathrm{C}$ prior to the deposition of AlN heteroepitaxial layers on sapphire, while the nucleation layer was eliminated for the growth of homoepilayers. The AlN epilayers were grown at $1300{ }^{\circ} \mathrm{C}$ and the V/III ratio employed was the same for all samples and was about 50. Trimethylaluminum and ammonia were used as $\mathrm{Al}$ and $\mathrm{N}$ sources, respectively. The PL spectra were measured with a DUV laser spectroscopy system that consists of frequency quadrupled $100 \mathrm{fs}$ Ti-sapphire laser with $76 \mathrm{MHz}$ repetition rate and photon energy set around $6.30 \mathrm{eV}$. Since the optical absorption coefficient in AlN for the above band gap excitation is typically
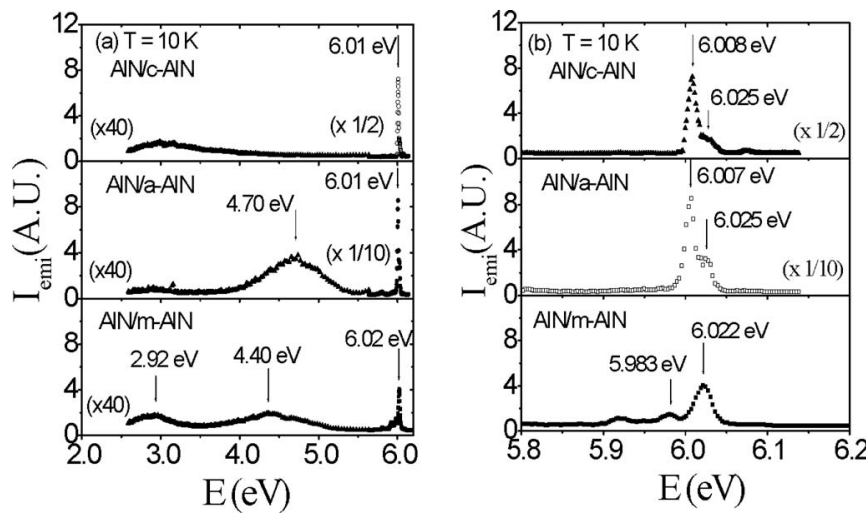

FIG. 1. Low temperature $(10 \mathrm{~K}) \mathrm{PL}$ spectra of $c$-plane, $a$-plane, and $m$-plane AlN homoepilayers measured in a (a) wide spectral range from 2 to $6.2 \mathrm{eV}$ and (b) narrow spectral range from 5.8 to $6.2 \mathrm{eV}$. 


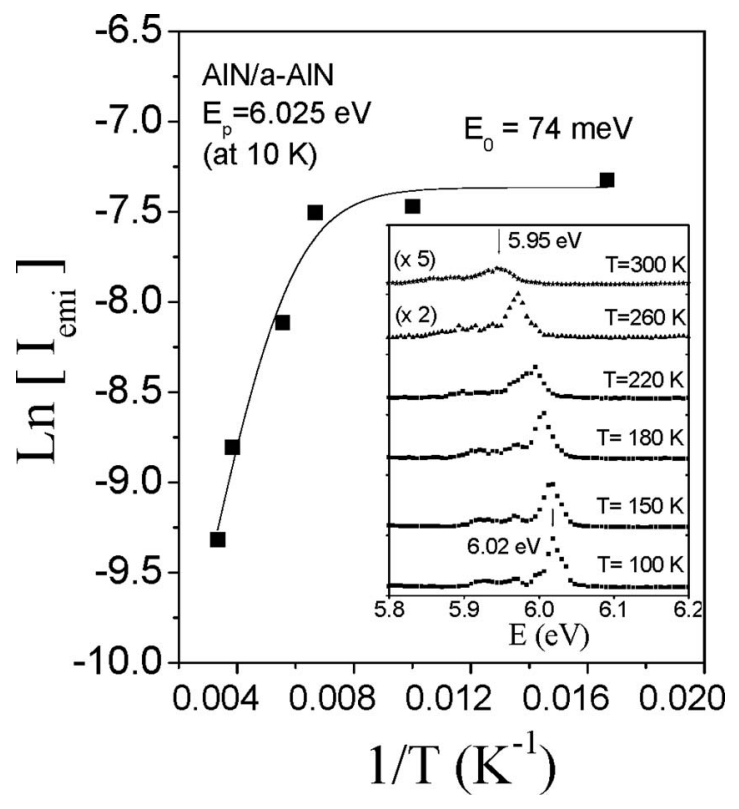

FIG. 2. Arrhenious plot of the PL intensity $\left[\operatorname{Ln}\left(I_{\mathrm{emi}}\right)\right.$ vs $\left.1 / T\right]$ of FX transition $(6.025 \mathrm{eV}$ at $10 \mathrm{~K})$ for an $a$-plane AlN homoepilayer. The solid line is the least squares fit of data with Eq. (1). The inset shows the temperature evolution of the band-edge emission between 100 and $300 \mathrm{~K}$.

$>10^{5} / \mathrm{cm},{ }^{14}$ such an excitation scheme provides a typical optical absorption depth of $<0.1 \mu \mathrm{m}$, which is much smaller than the epilayer thickness $(1 \mu \mathrm{m})$. Hence the measured PL emission was purely from the epilayer.

Figure 1 compares the low temperature (10 K) PL spectra of AlN homoepilayers with three different orientations (a-plane, $c$-plane and $m$-plane) (a) in a broad spectral range from 2.0 to $6.2 \mathrm{eV}$ and (b) in a narrower spectral range from 5.8 to $6.2 \mathrm{eV}$. We observed weak and deep impurity related emission bands centered around $2.9 \mathrm{eV}$ and $4.4 \mathrm{eV}$ from the $m$-plane sample related to $\mathrm{O}$ impurities ${ }^{15}$ and at $4.70 \mathrm{eV}$ in $a$-plane sample related to $\mathrm{Al}$ vacancy complex with one negative charge, ${ }^{16}\left(V_{\mathrm{Al}^{-}} \text {-complex }\right)^{1-}$. However, the intensities of these impurity transitions are less than $1 \%$ of the corresponding band-edge emissions, which confirms the high optical quality of our AlN homoepilayers.

Figure 1(b) shows the dominant peak at 6.008 (6.007) eV in $c$-plane ( $a$-plane) homoepilayer due to the donor bound exciton transition $\left(I_{2}\right)$, while the $6.025 \mathrm{eV}$ peak due to the free exciton transition (FX). $I_{2}$ is about $17 \mathrm{meV}$ below FX, which is the binding energy of the donor bound excitons in AlN and agrees well with the previously reported value. Figure 2 shows the Arrhenius plot of the PL intensity of the high energy peak $(6.025 \mathrm{eV}$ at $10 \mathrm{~K})$ measured in $a$-plane AlN homoepilayer. The inset of Fig. 2 shows the temperature dependent PL spectra. The solid line is the least squares fit of data with the equation

$$
I(T)=\frac{I_{0}}{1+C e^{-E_{0} / K_{B} T}} .
$$

The fitted value of $E_{0}$ is $74 \mathrm{meV}$, which is close to the previously reported value of $78 \mathrm{meV}$ for the binding energy of FX in $\mathrm{AlN} / \mathrm{Al}_{2} \mathrm{O}_{3}$ and further corroborates our assignment of a FX transition for the $6.025 \mathrm{eV}$ peak. In $m$-plane homoepilayers, there is only one dominant FX transition at $6.022 \mathrm{eV}$ and no observable $I_{2}$ transition. The $5.983 \mathrm{eV}$ peak in this sample is $4 \mathrm{LO}$ of excitation laser. We thus conclude that all AlN homoepilayers (c-plane, $a$-plane, and $m$-plane) have almost identical FX peak positions (varying only slightly between 6.022 and $6.025 \mathrm{eV}$ ) at $10 \mathrm{~K}$. no $I_{2}$ emission is visible in $m$-plane AlN. This implies a smaller amount of unintentional donor impurity incorporation during epigrowth into $m$-plane films than $a$-plane and $c$-plane films. This observation is consistent with a previous study that revealed that the oxygen incorporation on $m$-plane is $\frac{1}{5}$ of that on $c$-plane of the sublimation grown AlN bulk crystals. ${ }^{17}$

The PL properties of AlN homoepilayers and heteroepilayers grown in different orientations are summarized in Table I for comparison. The near band-edge emission spectra measured in all $a$-plane, $c$-plane, and $m$-plane AlN homoepilayers at $300 \mathrm{~K}$ exhibit only one peak at an energy position of $5.961 \mathrm{eV}$ due to the FX transition, which lines up exactly with the FX peak position in AlN bulk crystals. All homoepilayers, therefore, are nearly stress free and the band gap of strain free AlN is thus $(6.025+0.074) \mathrm{eV}=6.099 \mathrm{eV}$ at $10 \mathrm{~K}$ and $(5.961+0.074) \mathrm{eV}=6.035 \mathrm{eV}$ at $300 \mathrm{~K}$. This conclusion is further corroborated by the x-ray (XRD) measurements of the lattice parameters. For example, the spectral peak positions of $\theta / 2 \theta$ scans of both the symmetric (002) and asymmetric (102) reflection peaks of a $c$-plane AlN homoepilayer and AlN bulk substrate exactly line up revealing an in-plane lattice constant of $a=3.112 \AA$, which also indi-

TABLE I. Comparison of PL emission properties of $a$-plane, $c$-plane, and $m$-plane AlN homoepilayers, $c-\mathrm{AlN} / c-\mathrm{Al}_{2} \mathrm{O}_{3}$, and $a-\mathrm{AlN} / r-\mathrm{Al}_{2} \mathrm{O}_{3}$ heteroepilayers including emission peak position $\left(E_{p}\right)$, peak intensity $\left(I_{p}\right)$, full width at half maximum (FWHM), and binding energy $\left(E_{0}\right)$.

\begin{tabular}{|c|c|c|c|c|c|c|c|}
\hline & & \multirow{2}{*}{$\begin{array}{c}\text { Transition } \\
\text { type }\end{array}$} & \multicolumn{4}{|l|}{$T=10 \mathrm{~K}$} & \multirow{2}{*}{$\begin{array}{c}300 \mathrm{~K} \\
E_{p}(\mathrm{eV})\end{array}$} \\
\hline & & & $E_{p}(\mathrm{eV})$ & $I_{p}($ a.u. $)$ & FWHM (meV) & $E_{0}(\mathrm{meV})$ & \\
\hline \multirow{7}{*}{ Homoepilayers } & & $\mathrm{FX}$ & 6.025 & 24 & 16.0 & 74 & 5.961 \\
\hline & $c$ plane & $I_{2}$ & 6.008 & 136 & 13.1 & 17 & \\
\hline & $a$ plane & $\mathrm{FX}$ & 6.025 & 320 & 13.0 & 74 & 5.961 \\
\hline & & $I_{2}$ & 6.007 & 820 & 11.6 & 18 & \\
\hline & $m$ plane & FX & 6.022 & 35 & 21.0 & $\cdots$ & 5.961 \\
\hline & & FX & 6.063 & 89 & 22 & 78 & 5.984 \\
\hline & & $I_{2}$ & 6.045 & 63 & 19.2 & 18 & \\
\hline \multirow{2}{*}{$\begin{array}{l}\text { Heteroepilayers } \\
\quad \text { (Sapphire) }\end{array}$} & & FX & 6.030 & 38 & 33 & $\cdots$ & 5.956 \\
\hline & & $I_{2}$ & 6.013 & 23 & 20 & 17 & \\
\hline AlN bulk & $c$ plane & $\mathrm{FX}$ & $\cdots$ & $\cdots$ & $\cdots$ & $\ldots$ & 5.961 \\
\hline
\end{tabular}



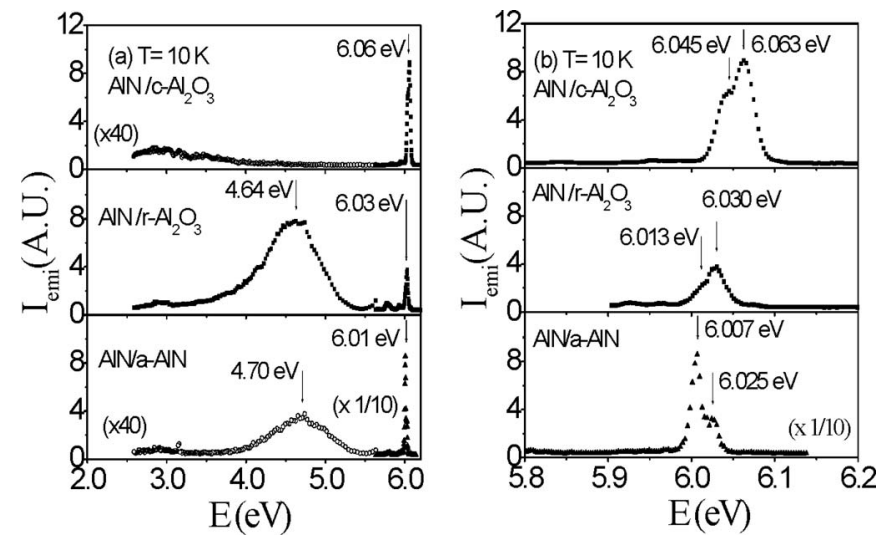

FIG. 3. Low temperature $(10 \mathrm{~K}) \mathrm{PL}$ spectra of $c-\mathrm{AlN} / c-\mathrm{Al}_{2} \mathrm{O}_{3}$, $a$ - $\mathrm{AlN} / r-\mathrm{Al}_{2} \mathrm{O}_{3}$ and $a$-plane $\mathrm{AlN}$ homoepilayers measured in a (a) wide spectral range from 2 to $6.2 \mathrm{eV}$ and (b) narrow spectral range from 5.8 to $6.2 \mathrm{eV}$.

cate that the AlN homeepilayer is perfectly lattice matched to the AlN bulk substrate and is almost strain free. ${ }^{18}$

The band-edge emission peak intensity ratio for $a$-plane, $c$-plane, and $m$-plane AlN homoepilayers is approximately 32:5:1. Due to the unique band structure of AlN near the $\Gamma$ point, the PL intensity in $a$-plane AIN is enhanced by the fact that AlN exhibits a maximum emission in the directions perpendicular to the $c$ direction (or in the $\mathbf{E} / / \mathbf{c}$ measurement geometry). ${ }^{19}$ In our measurement, the $c$ direction of $a$-plane AlN lies in the substrate plane so that the maximum emitted light propagation direction is normal to the surface. Thus, optoelectronic devices with $a$-plane homoepilayers in their active region can exploit optimum quantum efficiency.

Figure 3 shows the $10 \mathrm{~K} \mathrm{PL}$ spectra of a $c$-plane AlN epilayer grown on $c$-sapphire and $a$-plane AlN epilayers grown on $r$-plane sapphire and also on $a$-plane bulk AlN (a) covering a broad spectral range from 2.0 to $6.2 \mathrm{eV}$ and (b) in a small spectral range from 5.8 to $6.2 \mathrm{eV}$. The near bandedge emission spectra of an AlN epilayer grown on $c$-plane ( $r$-plane) sapphire has two peaks at 6.063 (6.030) and 6.045 (6.013) $\mathrm{eV}$, corresponding to the recombination of FX and $I_{2} .{ }^{16}$ Clearly the FX emission peak in $a$-plane AIN heteroepilayer is about $33 \mathrm{meV}$ lower than that in $c$-AlN heteroepilayer. This implies that the strain induced by lattice mismatch between AlN and sapphire substrate is almost relaxed by simply switching the growth to $r$-plane. However, compared with homoepilayers, the unique band structure induced enhancement in the band-edge emission intensity is absent in $a$-plane AlN heteroepilayers due to the presence of high dislocation density because the growth technology for $a$-plane AlN epilayers is still far from mature. This interpretation is consistent with three other observations: (1) the FX emission linewidth is narrower in $c$-plane AlN heteroepilayers than that in $a$-plane hetero-epilayers; (2) the presence of a much higher concentration of $\mathrm{Al}$ vacancies in $a$-plane AlN heteroepilayers; (3) the full width at half maximum of XRD rocking curve of the asymmetric (102) reflection peak of $c$-plane AlN heteroepilayers (400 arc sec) is much narrower than that of the (110) reflection peak of $a$-plane AlN heteroepilayers. (940 arc sec).

We can also make a comparison between emission spectra of $a$-plane homoepilayers and heteroepilayers. As seen in Fig. 3, the near band-edge spectrum of $a$-plane AlN ho- moepilayer exhibits an emission peak at $6.025 \mathrm{eV}$ due to the FX recombination, which is only about $5 \mathrm{meV}$ below that in $a$-plane AlN heteroepilayer, which reiterates the fact that the strain induced by the use of a foreign substrate is very small in $a$-plane AlN. The $a$-plane homoepilayer has an emission intensity that is at least one order of magnitude higher and a much narrower emission linewidth compared to those of $a$-plane heteroepilayers, although no optimization processes for homoepilayer growth was carried out due to the limited supply of AlN bulk substrates. The results reaffirm the higher crystalline and optical qualities of homoepilayers over heteroepilayers.

In summary, we have carried out MOCVD growth and investigated the optical properties of AlN homoepilayers and heteroepilayers. Our results indicate that homoepilayers are nearly stress free and possess an identical band gap of about $6.099(6.035) \mathrm{eV}$ at $10(300) \mathrm{K}$. The stress on $a$-plane AlN heteroepilayers grown on $r$-plane sapphires is very small. Due to the unique band structure of AlN, we believe that $a$-plane AlN homoepilayers have the potential to provide orders of magnitude improvement in the performance of new generation DUV photonic devices, especially surface emitting devices.

The epigrowth and optical study works were supported by DOE (Grant No. DE-FG 03-96ER45604) and the AlN bulk crystal growth work was supported by NSF (Grant No. DMR 0415116). J. Y. L. and H. X. J. gratefully acknowledge the support of the Linda Whitacre and Edward Whitacre Endowment through the AT \& T Foundation.

${ }^{1}$ K. Dovidenko, S. Oktyabrsky, J. Narayan, and M. Razeghi, J. Appl. Phys. 79, 2439 (1996).

${ }^{2}$ Y. Taniyasu, M. Kasu, and T. Makomoto, Nature (London) 441, 325 (2006).

${ }^{3}$ J. Li, Z. Y. Fan, R. Dahal, M. L. Nakarmi, J. Y. Lin, and H. X. Jiang, Appl. Phys. Lett. 89, 213510 (2006).

${ }^{4}$ R. Dahal, T. M. Al Tahtamouni, J. Y. Lin, and H. X. Jiang, Appl. Phys. Lett. 91, 243503 (2007).

${ }^{5}$ M. A. Khan, R. A. Skogman, J. M. Van Hove, S. Krishnankutty, and R. M. Kolbas, Appl. Phys. Lett. 56, 1257 (1990).

${ }^{6}$ S. Nakamura, T. Mukai, M. Senoh, S. Nagahama, and N. Iwasa, J. Appl. Phys. 74, 3911 (1993).

${ }^{7}$ F. A. Ponce and D. P. Bour, Nature (London) 386, 351 (1997).

${ }^{8}$ F. Bernardini, V. Fiorentini, and D. Vanderbilt, Phys. Rev. B 56, R10024 (1997)

${ }^{9}$ H. M. Ng, Appl. Phys. Lett. 80, 4369 (2002).

${ }^{10}$ T. M. Al Tahtamouni, A. Sedhain, J. Y. Lin, and H. X. Jiang, Appl. Phys. Lett. 90, 221105 (2007).

${ }^{11}$ T. J. Baker, B. A. Haskell, F. Wu, P. T. Fini, J. S. Speck, and S. Nakamura, Jpn. J. Appl. Phys., Part 2 44, L920 (2005).

${ }^{12}$ Z. Gu, L. Du, J. H. Edgar, N. Nepal, J. Y. Lin, H. X. Jiang, and R. Witt, J. Cryst. Growth 297, 105 (2006).

${ }^{13}$ D. Zhang, J. H. Edgar, B. Liu, H. E. Huey, H. X. Jiang, J. Y. Lin, M. Kuball, F. Mogal, J. Chaudhuri, and Z. Rek, J. Cryst. Growth 262, 89 (2004).

${ }^{14}$ P. B. Perry and R. F. Rutz, Appl. Phys. Lett. 33, 319 (1978).

${ }^{15}$ M. Bickermann, B. M. Epelbaum, and A. Winnacker, Phys. Status Solidi C 0, 7 (2003)

${ }^{16}$ N. Nepal, M. L. Nakarmi, J. Y. Lin, and H. X. Jiang, Appl. Phys. Lett. 89, 092107 (2006).

${ }^{17}$ P. Lu, R. Collazo, R. Dalmau, X. Li, J. Tweedie, and Z. Sitar, MRS Symposia No. 1040E (Materials Research Society, Pittsburgh, 2007).

${ }^{18}$ B. N. Pantha, N. Nepal, T. M. Al Tahtamouni, M. L. Nakarmi, J. Y. Lin, and H. X. Jiang, Appl. Phys. Lett. 91, 121117 (2007).

${ }^{19}$ K. B. Nam, J. Li, M. L. Nakarmi, J. Y. Lin, and H. X. Jiang, Appl. Phys. Lett. 84, 5264 (2004). 